\title{
DENSE SUBMODULES
}

\section{T. J. HEAD ${ }^{1}$}

J. M. Irwin suggested [1] the problem of determining those primary abelian groups $G$ for which the kernel of each homomorphism of $G$ onto a divisible group contains a basic subgroup of $G$. This question is answered in the corollary below. The basic references for this paper are [2] and [3]. All groups considered are modules over a discrete valuation ring $R$, i.e. over a principal ideal ring with a unique maximal ideal $(p)$. We let $Q$ be the additive group of the quotient field of $R$. For the purposes of this discussion it is convenient to define:

Definition. A submodule $K$ of an $R$-module $G$ is called a dense submodule of $G$ if $G / K$ is divisible.

If $G$ has no elements of infinite height, then a submodule $K$ of $G$ is dense in the $p$-adic topology if and only if $K$ is a dense submodule as just defined.

The proof of the following theorem is presented in the three propositions below.

Theorem. $A$ module $G$ over a discrete valuation ring $R$ has the property that every dense submodule of $G$ contains a basic submodule of $G$ if and only if either the torsion submodule of $G$ is divisible or $G$ is the direct sum of a bounded module and a free module of finite rank.

CoROLlaRY. A primary abelian group $G$ has the property that every dense subgroup of $G$ contains a basic subgroup of $G$ if and only if either $G$ is divisible or $G$ is of bounded order.

A submodule $N$ of $G$ is neat in $G$ in case $N \cap p G=p N$. (This may be compared with the definition: a submodule $S$ of $G$ is pure in $G$ in case $S \cap p^{k} G=p^{k} S$, for all integers $k \geqq 0$.) Observe that any pure submodule is neat. On the other hand, the cyclic submodule generated by an element of height zero is always neat, but it may not be pure. If $G$ is either torsion-free or divisible, then every neat submodule is pure. In particular, a neat submodule of a divisible module is divisible. If $G$ contains submodules $H$ and $N$ where $H \supset N$ and $N$ is neat in $G$ then if $H / N$ is neat in $G / N$ it follows that $H$ is neat in $G$. Additional information on neat submodules can be found in [2].

Received by the editors September 14, 1960 and, in revised form, December 22, 1960 and March 6, 1961.

${ }^{1}$ Part of the work done on this paper was done while the author was attending an RPPTT Institute sponsored by the NSF at the University of Oklahoma. The author is thankful to the referee for extensive suggestions and corrections. 
Lemma 1. If the torsion submodule of an $R$-module $G$ is divisible then every neat submodule of $G$ is pure.

Proof. Let $T$ be the torsion submodule of $G$, and let $N$ be neat in $G$. Now the torsion submodule $S$ of $N$ is neat in $N$. Since $N$ is neat in $G, S$ is neat in $G$; a fortiori, $S$ is neat in $T$. Then $S$ is divisible and $N=S \oplus H$ where $H$ is torsion-free. There is a decomposition $G=T \oplus F$ such that $H \subset F$. Then $H$ is neat in $F$ and since $F$ is torsion-free $H$ is pure in $F$. Finally, $N=S \oplus H$ is pure in $T \oplus F=G$.

Proposition 1. If the torsion submodule of the $R$-module $G$ is divisible then each dense submodule of $G$ contains a basic submodule of $G$.

Proof. Let the torsion submodule $T$ of $G$ be divisible and let $K$ be a dense submodule of $G$. Let $M$ be a pure submodule of $G$ maximal with respect to inclusion in $K$. The torsion submodule $T^{\prime}$ of $M$ is pure in $T$ and is therefore divisible. Thus $M=T^{\prime} \oplus F^{\prime}$ where $T^{\prime}$ is divisible and $F^{\prime}$ is torsion-free. Also $G=T \oplus F$ for some $F \supset F^{\prime}$ and $F^{\prime}$ is pure in $F$. Thus the torsion submodule of $G / M$ is isomorphic to $T / T^{\prime}$ which is divisible. By Lemma 1 , if $K / M$ contained an element of zero height in $G / M$ then the submodule it generated would be pure in $G / M$. This would contradict the maximality condition on $M$. Thus for each $k \in K$ there is a $g \in G$ and an $m \in M$ such that $k=p g+m$. Since $G / K$ is divisible we have for any $g \in G$ elements $g_{1} \in G$ and $k_{1} \in K$ such that $g=p g_{1}+k_{1}$. Since also $k_{1}=p g_{2}+m$ for $g_{2} \in G, m \in M$, we have $g=p\left(g_{1}+g_{2}\right)+m$. Thus $G / M$ is divisible and $M$ is a pure dense submodule contained in $K$. Then for any basic submodule $B$ of $M, B$ is basic in $G$ and is contained in $K$.

Proposition 2. For a nondivisible $R$-module $G$, the following three conditions are equivalent:

(i) G contains no proper dense submodule;

(ii) $G$ contains no proper basic submodule;

(iii) $G=T \oplus F$ where $T$ is a bounded module and $F$ is a free module of finite rank.

Proof. (i) implies (ii): Trivial.

(ii) implies (iii): Let $B$ be a basic submodule of $G$. Since $G$ is not divisible, $B \neq 0$. Since $B$ is not proper, $B=G$. Hence $G$ is the direct sum of cyclic modules, and so $B=T \oplus F$ where $T$ is the direct sum of cyclic torsion modules and $F$ is free. If $F$ had infinite rank, there would exist an epimorphism $f: F \rightarrow Q$, with kernel $K$. $K$ would be pure in $G$ since $Q$ is torsion-free. But then $T \oplus K$ would be a proper basic submodule of $G$. Thus $F$ has finite rank. If $T$ were not bounded, $T$ would contain a proper submodule $L$ basic in $T$ and $L \oplus F$ would 
be a proper basic submodule of $G$. Thus $T$ is of bounded order.

(iii) implies (i): Let $h$ be a homomorphism of $T \oplus F$ into $Q / R$. It is easy to see that there is an integer $k \geqq 0$ such that $p^{k} h(T \oplus F)=0$. Hence $h$ is not an epimorphism. Then $T \oplus F$ does not possess $Q / R$ as a homomorphic image and therefore contains no proper dense submodule.

Lemмa 2. If $N$ is a neat submodule of an $R$-module $G$ and if $N$ contains a basic submodule of $G$ then $N$ is pure in $G$.

Proof. Let $B \subset N \subset G$ with $B$ a basic submodule of $G$ and $N$ neat in $G$. Then $N / B$ is neat in $G / B$ and since $G / B$ is divisible, $N / B$ is pure in $G / B$. By the purity of $B, N$ is pure in $G$.

Proposition 3. If the torsion module of an $R$-module $G$ is not divisible and if $G$ is not the direct sum of a bounded module and a free module of finite rank then $G$ contains a neat dense submodule $N$ such that $N$ contains no basic submodule of $G$ and $G / N \cong Q / R$.

Proof. Let $G$ satisfy the condition of the proposition. Then $G$ contains a submodule $G_{1}$ such that $G=\{x\} \oplus G_{1}$ where $x \neq 0$ is of some finite order $p^{n}$. Either $G_{1}$ is divisible or $G_{1}$ contains, by Proposition 2, a proper basic submodule of itself. In either case $G_{1}$ contains a pure submodule $P$ such that $D=G_{1} / P$ is a divisible module of rank 1 . Then $G / P \cong\{x\} \oplus D$. Let $y$ be an element of $D$ such that $p^{n} y \neq 0$. Then $\{x+y\}$ is neat in $\{x\} \oplus D$ but it is not pure since $p^{n} y=p^{n}(x+y)$ $\in\{x+y\} \cap D$. Let $N$ be the complete inverse image in $G$ of $\{x+y\}$ with respect to the homomorphism of $G$ onto $\{x\} \oplus D$. Then since $P$ is pure we conclude that $N$ is neat but not pure in $G$. Further, $G / N \cong(\{x\} \oplus D) /\{x+y\} \cong Q / R$. Finally, by Lemma $2, N$ contains no basic submodule of $G$.

\section{Bibliography}

1. J. M. Irwin, High subgroups of abelian torsion groups, Doctoral thesis, University of Kansas, 1960.

2. L. Fuchs, Abelian groups, Publ. House of the Hungarian Acad. of Sci., Budapest, 1958. 1954.

3. I. Kaplansky, Infinite abelian groups, Univ. of Michigan Press, Ann Arbor,

WASHBURN UNIVERSITY 